\title{
Insights into the diversification of subclade IVa bHLH transcription factors in Fabaceae
}

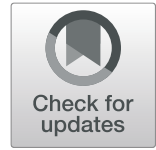

Hayato Suzuki ${ }^{1}$, Hikaru Seki ${ }^{1,2}$ and Toshiya Muranaka ${ }^{1,2^{*}}$ (D)

\begin{abstract}
Background: Fabaceae plants appear to contain larger numbers of subclade IVa basic-helix-loop-helix (bHLH) transcription factors than other plant families, and some members of this subclade have been identified as saponin biosynthesis regulators. We aimed to systematically elucidate the diversification of this subclade and obtain insights into the evolutionary history of saponin biosynthesis regulation in Fabaceae.

Results: In this study, we collected sequences of subclade IVa bHLH proteins from 40 species, including fabids and other plants, and found greater numbers of subclade IVa bHLHs in Fabaceae. We confirmed conservation of the bHLH domain, C-terminal ACT-like domain, and exon-intron organisation among almost all subclade IVa members in model legumes, supporting the results of our classification. Phylogenetic tree-based classification of subclade IVa revealed the presence of three different groups. Interestingly, most Fabaceae subclade IVa bHLHs fell into group 1, which contained all legume saponin biosynthesis regulators identified to date. These observations support the cooccurrence and Fabaceae-specific diversification of saponin biosynthesis regulators. Comparing the expression of orthologous genes in Glycine max, Medicago truncatula, and Lotus japonicus, orthologues of MTTSAR1 (the first identified soyasaponin biosynthesis regulatory transcription factor) were not expressed in the same tissues, suggesting that group 1 members have gained different expression patterns and contributions to saponin biosynthesis during their duplication and divergence. On the other hand, groups 2 and 3 possessed fewer members, and their phylogenetic relationships and expression patterns were highly conserved, indicating that their activities may be conserved across Fabaceae.

Conclusions: This study suggests subdivision and diversification of subclade IVa bHLHs in Fabaceae plants. The results will be useful for candidate selection of unidentified saponin biosynthesis regulators. Furthermore, the functions of groups 2 and 3 members are interesting targets for clarifying the evolution of subclade IVa bHLH transcription factors in Fabaceae.
\end{abstract}

Keywords: Fabaceae, Triterpene saponin, bHLH, Transcriptional regulation, Classification

\footnotetext{
* Correspondence: muranaka@bio.eng.osaka-u.ac.jp

'Department of Biotechnology, Graduate School of Engineering, Osaka

University, 2-1 Yamadaoka, Suita, Osaka 565-0871, Japan

${ }^{2}$ Industrial Biotechnology Initiative Division, Institute for Open and

Transdisciplinary Research Initiatives, Osaka University, 2-1 Yamadaoka, Suita,

Osaka 565-0871, Japan
}

(c) The Author(s). 2021 Open Access This article is licensed under a Creative Commons Attribution 4.0 International License, which permits use, sharing, adaptation, distribution and reproduction in any medium or format, as long as you give appropriate credit to the original author(s) and the source, provide a link to the Creative Commons licence, and indicate if changes were made. The images or other third party material in this article are included in the article's Creative Commons licence, unless indicated otherwise in a credit line to the material. If material is not included in the article's Creative Commons licence and your intended use is not permitted by statutory regulation or exceeds the permitted use, you will need to obtain permission directly from the copyright holder. To view a copy of this licence, visit http://creativecommons.org/licenses/by/4.0/ The Creative Commons Public Domain Dedication waiver (http://creativecommons.org/publicdomain/zero/1.0/) applies to the data made available in this article, unless otherwise stated in a credit line to the data. 


\section{Background}

Triterpene saponins are a group of plant specialised (secondary) metabolites found widely across the plant kingdom [1]. Triterpenes have been intensively studied in terms of their biosynthesis [2], transcriptional regulation [3-5], and bioactivities [6-8]. The monocotyledonous plants in the genus Avena produce antifungal saponins, known as avenacins in the roots for protection against take-all disease [9]. Saponins produced by Barbarea vulgaris (Brassicaceae) show antifeedant activity against insect herbivores [10]. Fabaceae (Leguminosae) plants produce structurally diverse triterpenes, including hemolytic saponins in Medicago truncatula, betulinic acid in Lotus japonicus, and glycyrrhizin in Glycyrrhiza uralensis, as well as a common group called soyasaponins [11-15]. Leguminous triterpenes affect symbiotic nodulation, as transgenic $M$. truncatula with elevated saponin content showed enhanced nodulation [16] and $L$. japonicus mutants lacking lupeol (the precursor of betulinic acid) showed a phenotype of rapid nodulation [17].

Basic-helix-loop-helix (bHLH) transcription factors are one of the largest families of plant transcription factors, and are classified into approximately 25 subclades based on sequence homology within the bHLH domain and other shared protein domains [18, 19]. Land plants have acquired more bHLH genes than animals, chlorophytes, or red algae [19], and some subclades evolved to regulate plant specialised metabolism [5]. Subclade IVa is a good example of such regulation, as it represents conserved transcriptional regulation of methyl jasmonate (MeJA)-mediated metabolic processes in plants [5]. TRITERPENE SAPONIN BIOSYNTHESIS ACTIVATING REGULATOR1 (MtTSAR1) upregulates the soyasaponin pathway in M. truncatula [20]. MtTSARs 2 and 3 are factors that activate hemolytic saponin accumulation, with differences in tissue specificity [20, 21]. We recently identified GubHLH3 as a positive regulator of soyasaponin biosynthesis in $G$. uralensis [22], and this protein is closely related to MtTSAR2 but not MtTSAR1. This finding hints at the evolutionary history of Fabaceae subclade IVa bHLHs. Chenopodium quinoa (Amaranthaceae) seeds accumulate saponins with similar structures to the hemolytic saponins of M. truncatula. Mutations in CqTSAR-like1 (CqTSARL1) were identified as a major factor affecting differences in the saponin accumulation pattern between saponin-producing and saponin-lacking ecotypes [23]. In Catharanthus roseus (Apocynaceae), bHLH iridoid synthesis 1 (CrBIS1) and CrBIS2 were found to positively regulate the biosynthesis pathway for the iridoid branch of monoterpenoid indole alkaloids (MIAs) [24, 25]. Interestingly, the functions of MtTSARs and CrBIS1 were shown to be interchangeable through heterologous expression of MtTSARs in C. roseus and CrBIS1 in $M$. truncatula [26]. In addition, production of both saponins and MIAs were commonly regulated by MeJA [5, 21, 24, 27].
Numerous studies have reported genome-wide identification and classification of bHLH factors in plants [18, 19, 2830]. Although the genomes of Arabidopsis thaliana and Oryza sativa possess four and six subclade IVa members, respectively [19], more than 30 subclade IVa bHLH genes were found in the genomes of Glycine max and $M$. truncatula $[21,28]$. This finding suggests that Fabaceae plants may have acquired a large number of subclade IVa members during the evolution of saponin biosynthesis.

In this study, we extensively explored subclade IVa bHLHs in fabids and showed that Fabaceae plants possess a large number of subclade IVa members, which were classified into three groups based on phylogenetic analysis. Group 1 had the greatest number of members, including MtTSARs and GubHLH3. Groups 2 and 3 contained fewer members, none of which were functionally-identified, but were obviously distinct from group 1 based on the tree and highly conserved among Fabaceae plants. We also performed in silico analysis to elucidate their structures and functions. This study will help to narrow down the candidates of unidentified saponin biosynthesis regulators and clarify the evolution of subclade IVa members in Fabaceae plants.

\section{Results \\ Large numbers of subclade IVa members in Fabaceae plants}

A total of 319 bHLH proteins and 33 subclade IVa members were identified previously in G. $\max$ [28]. We obtained 355 sequences of $G$. $\max$ bHLH proteins (Additional file 1: Table S1) using PlantTFDB [31]. Then, we assigned individual names to the novel members and re-selected subclade IVa members based on sequence similarity of the full-length proteins. Although five proteins (GmbHLH60-64) were designated as members of subclade IVa in a previous study [28], they had relatively long amino acid sequences (588-653 aa) and clustered more closely with bHLH proteins in subclade IIIf from $A$. thaliana on the phylogenetic tree (Additional file 3: Fig. S1). GmbHLH327, 329, 331, 334, 337, and 345 were newly assigned to subclade IVa based on Basic Local Alignment Search Tool (BLAST) search results. Finally, we identified 34 G. max subclade IVa bHLHs ranging in peptide length from 195 to 390 aa (Additional file 1: Table S1).

We collected sequences of all bHLH proteins from 40 plant species including $A$. thaliana, $C$. roseus, $C$. quinoa and various fabids (Additional file 1: Table S2). These proteins were used as queries for BLAST searches against the 4 and 34 subclade IVa bHLHs identified in A. thaliana and G. $\max$, and we thereby identified the subclade IVa members in each plant species (Additional file 2). Fabaceae plants possessed 61 to 355 bHLHs and 4 to 35 subclade IVa members, while species outside of Fabaceae had 94 to 250 bHLHs and 2 to 8 subclade IVa members (Table 1). Because genome 
Table 1 Numbers of total bHLH and subclade IVa genes

\begin{tabular}{|c|c|c|c|c|c|c|c|}
\hline ID & Species & bHLH & IVa & Group 1 & Group 2 & Group 3 & $\begin{array}{l}\% \\
\text { (IVa/bHLH) }\end{array}$ \\
\hline 01_Cl & Citrullus lanatus & 126 & 4 & 0 & 1 & 3 & 3.17 \\
\hline 02_Cm & Cucumis melo & 131 & 4 & 0 & 1 & 3 & 3.05 \\
\hline 03_Cs & Cucumis sativus & 130 & 4 & 0 & 1 & 3 & 3.08 \\
\hline 04_Ad & Arachis duranensis & 156 & 13 & 9 & 2 & 2 & 8.33 \\
\hline 05_Ah & Arachis hypogaea $^{a}$ & 72 & 4 & 4 & 0 & 0 & 5.56 \\
\hline 06_Ai & Arachis ipaensis & 160 & 11 & 7 & 2 & 2 & 6.88 \\
\hline 07_Cc & Cajanus cajan & 174 & 16 & 12 & 2 & 2 & 9.20 \\
\hline 08_Ca & Cicer arietinum & 140 & 14 & 10 & 2 & 2 & 10.0 \\
\hline 09_Gm & Glycine max & 355 & 34 & 26 & 4 & 4 & 9.58 \\
\hline 10_Gs & Glycine soja & 342 & 35 & 29 & 2 & 4 & 10.2 \\
\hline 11_Gu & Glycyrrhiza uralensis $^{\mathrm{b}}$ & 163 & 10 & 8 & 2 & 0 & 6.13 \\
\hline 12_Lj & Lotus japonicus & 152 & 15 & 10 & 2 & 3 & 9.87 \\
\hline 13_Mt & Medicago truncatula & 181 & 33 & 28 & 2 & 3 & 18.2 \\
\hline 14_Pv & Phaseolus vulgaris & 174 & 18 & 14 & 2 & 2 & 10.3 \\
\hline 15_Tp & Trifolium pratense & 147 & 15 & 11 & 2 & 2 & 10.2 \\
\hline 16_Va & Vigna angularis & 157 & 14 & 10 & 2 & 2 & 8.92 \\
\hline 17_Vr & Vigna radiata & 153 & 11 & 7 & 2 & 2 & 7.19 \\
\hline 18_Vu & Vigna unguiculata $^{a}$ & 61 & 4 & 4 & 0 & 0 & 6.56 \\
\hline 19_Cm & Castanea mollissima & 98 & 3 & 1 & 1 & 1 & 3.06 \\
\hline 20_Jr & Juglans regia & 125 & 6 & 2 & 2 & 2 & 4.80 \\
\hline 21_JC & Jatropha curcas & 113 & 4 & 2 & 1 & 1 & 3.54 \\
\hline 22_Me & Manihot esculenta & 184 & 5 & 2 & 2 & 1 & 2.72 \\
\hline 23_Rc & Ricinus communis & 121 & 3 & 1 & 1 & 1 & 2.48 \\
\hline 24_Lu & Linum usitatissimum & 195 & 7 & 2 & 2 & 3 & 3.59 \\
\hline 25_Pe & Populus euphratica & 178 & 4 & 1 & 2 & 1 & 2.25 \\
\hline 26_Pt & Populus trichocarpa & 201 & 4 & 1 & 2 & 1 & 1.99 \\
\hline 27_Sp & Salix purpurea & 219 & 4 & 1 & 3 & 0 & 1.83 \\
\hline $28 \_C s$ & Cannabis sativa & 99 & 2 & 1 & 1 & 0 & 2.02 \\
\hline 29_HI & Humulus lupulus & 103 & 5 & 1 & 2 & 2 & 4.85 \\
\hline 30_Mn & Morus notabilis & 116 & 3 & 1 & 1 & 1 & 2.59 \\
\hline 31_Zj & Ziziphus jujuba & 139 & 8 & 1 & 4 & 3 & 5.76 \\
\hline 32_Fv & Fragaria vesca & 112 & 3 & 1 & 1 & 1 & 2.68 \\
\hline 33_Fa & Fragaria $x$ ananassa & 94 & 3 & 1 & 1 & 1 & 3.19 \\
\hline 34_Md & Malus domestica & 250 & 8 & 2 & 2 & 4 & 3.20 \\
\hline 35_Pm & Prunus mume & 118 & 3 & 1 & 1 & 1 & 2.54 \\
\hline 36_Pp & Prunus persica & 129 & 3 & 1 & 1 & 1 & 2.33 \\
\hline 37_Pb & Pyrus bretschneideri & 197 & 8 & 1 & 4 & 3 & 4.06 \\
\hline 38_At & Arabidopsis thaliana & 153 & 4 & 0 & 4 & 0 & 2.61 \\
\hline 39_Cr & Catharanthus roseus & 96 & 5 & 0 & 5 & 0 & 5.21 \\
\hline 40_Cq & Chenopodium quinoa & 200 & 8 & 0 & 8 & 0 & 4.00 \\
\hline
\end{tabular}

Detailed classification of plants is summarised in Table S2. Fifteen Fabaceae plants are shown in bold. ${ }^{\mathrm{a}}$ Genomes had not been sequenced. ${ }^{\mathrm{b}}$ Draft genome database was used for sequence retrieval 


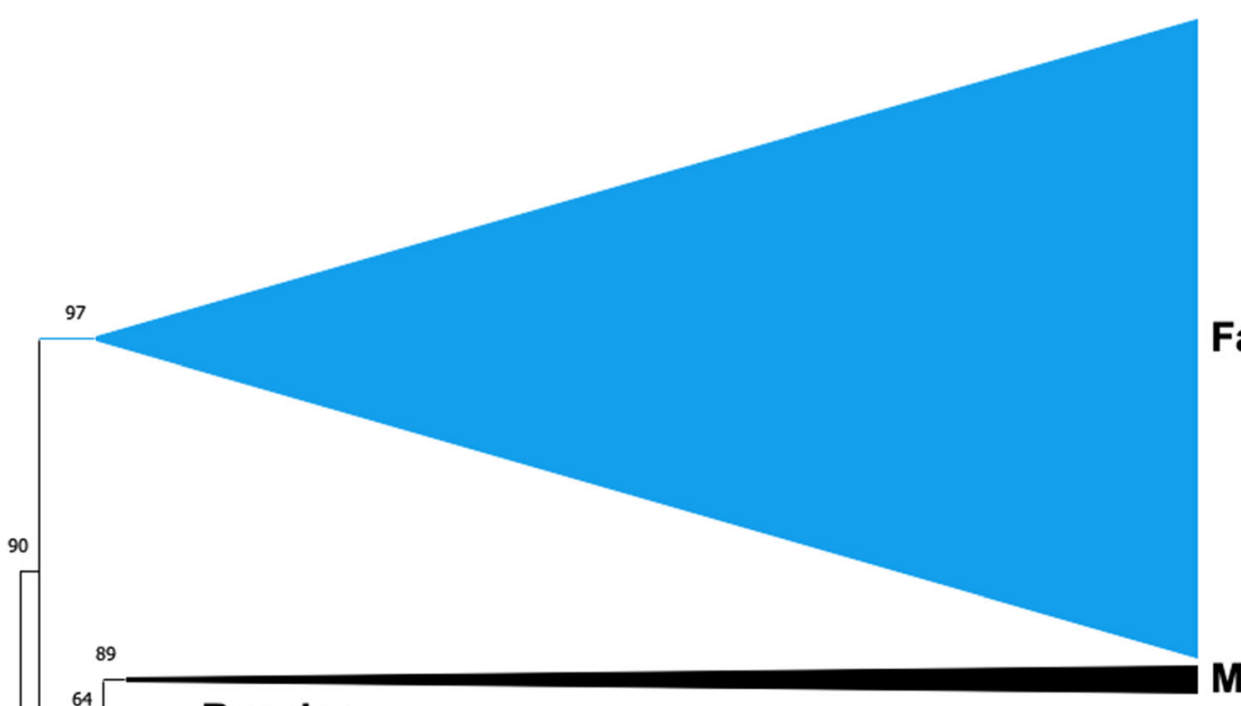

Fabales: group1

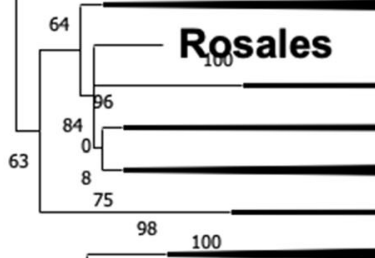

Malpighiales

Rosales

Fagales

Rosales

Malpighiales
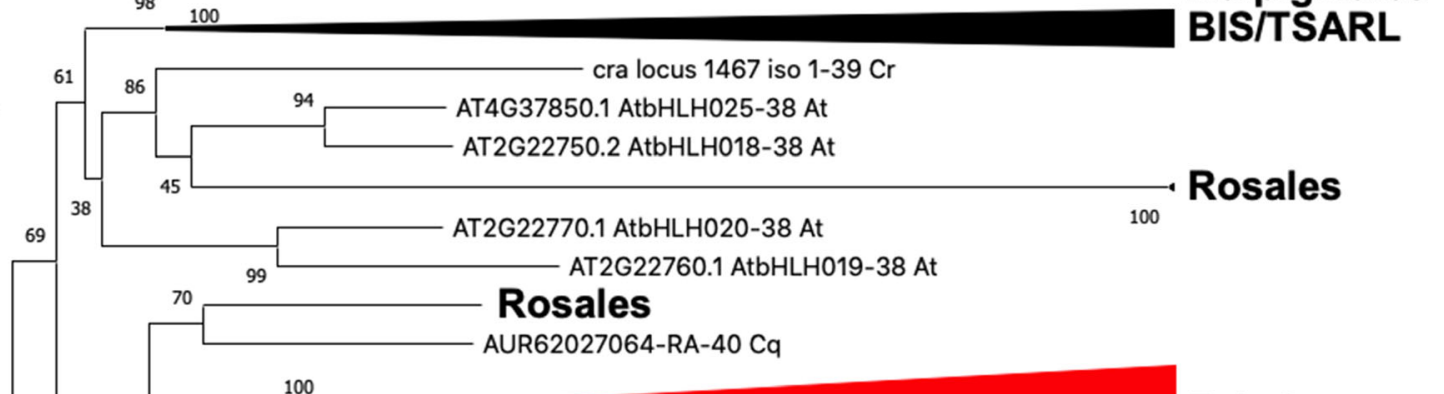

Fabales: group2

99

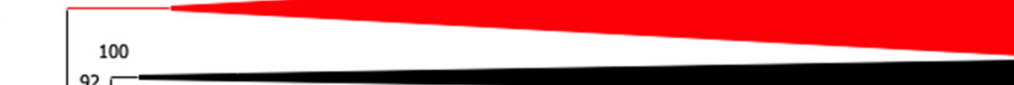

Rosales

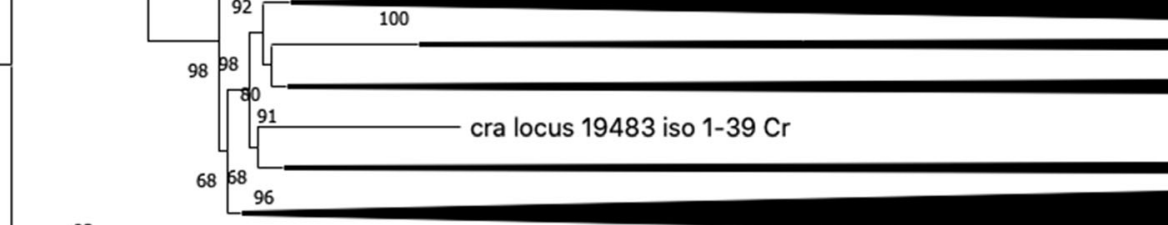

Cucurbitales

Rosales

Fagales

$93 \quad 92$

Malpighiales

$\square$

92

Malpighiales

(35

4

98

Rosales

Fagales

Rosales

36

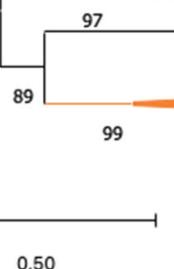

Rosales

Cucurbitales

Fabales: group3

Fig. 1 Phylogenetic tree of subclade IVa bHLH members in fabids. The approximately maximum-likelihood tree was built using FastTree and visualised with MEGA X. The local support values at each node were computed using 1000 resamples and the Shimodaira-Hasegawa test. Details are provided in Additional file 3: Fig. S2 


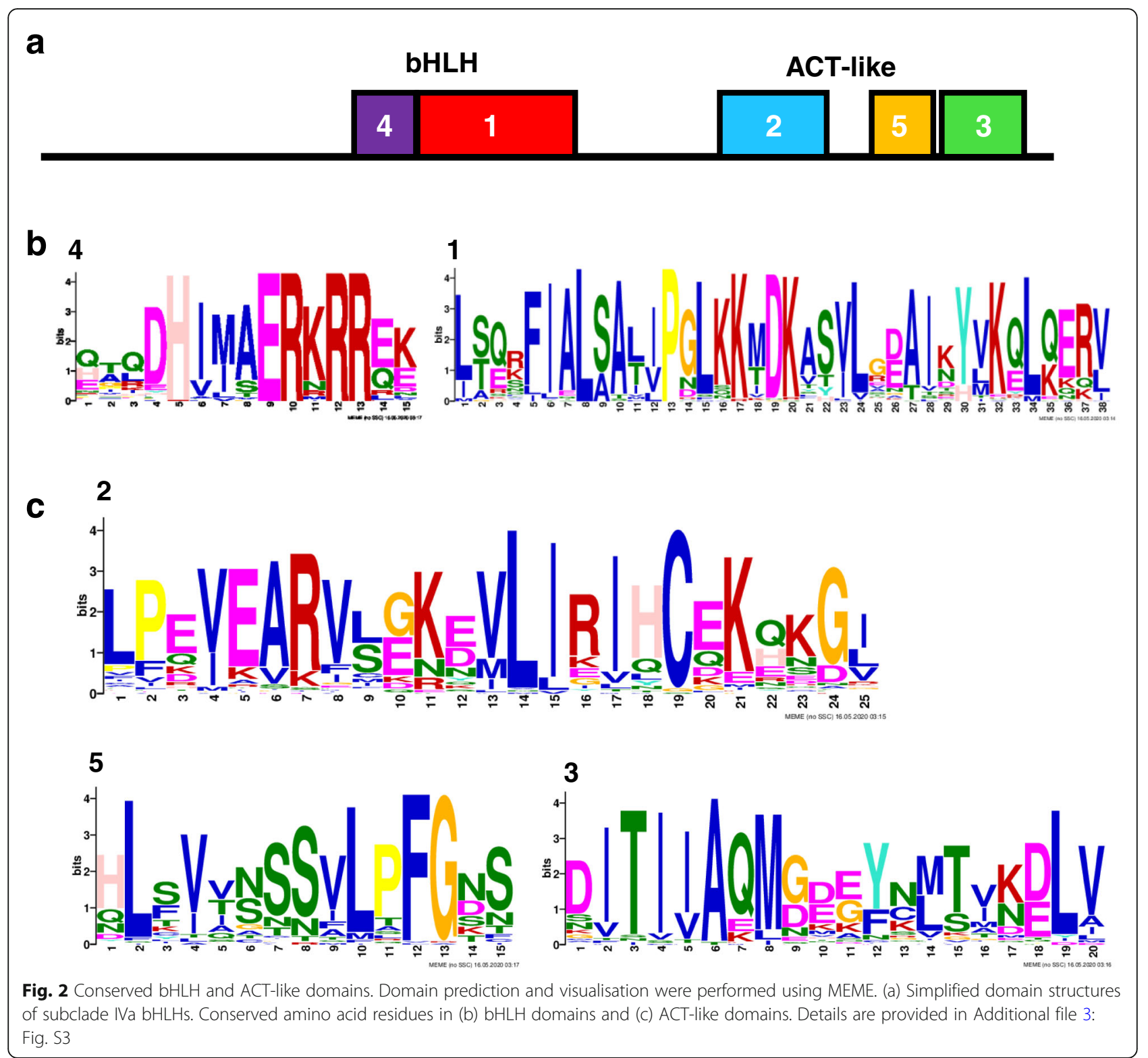

sequences of Arachis hypogaea and Vigna unguiculata were not used for the prediction in PlantTFDB, their bHLH sequences may not have all been collected. The percentage of subclade IVa genes relative to all bHLH genes was $5.56-18.2 \%$ and $1.82-5.76 \%$ in Fabaceae and non-Fabaceae fabids, respectively (Table 1 ). The genomes of Fabaceae contained significantly more subclade IVa bHLH genes than those of related plant families (Mann-Whitney U test, $U=329, p<10^{-9}$ ).

Three groups of subclade IVa bHLHs found in Fabaceae plants

To visualise the diversification of subclade IVa members in Fabaceae and other fabids, we constructed a phylogenetic tree using full-length sequences (Fig. 1,
Additional file 3: Fig. S2). Subclade IVa bHLHs were further classified into three groups. Most Fabaceae subclade IVa bHLHs were included in group 1 (Table 1), which contained all MtTSARs and GubHLH3. Groups 2 and 3 had limited numbers of members, but were highly conserved among Fabaceae plants (Additional file 3: Fig. S2).

\section{Conservation of bHLH and ACT-like domains and exon- intron structures}

As described in previous studies [16, 28], bHLHs have highly conserved protein domains with other members of the same subclade. Subclade IVa bHLHs contain a bHLH domain and C-terminal ACT-like domain; the basic region contacts cis-motifs on genomic DNA, while 
the HLH and ACT-like domains are involved in dimerisation [18, 25, 32, 33]. Using MEME algorithm [34], we searched for these conserved domains (Fig. 2, Additional file 3: Fig. S3) in 82 subclade IVa bHLHs of G. $\max , M$. truncatula, and L. japonicus (Additional file 1: Table S1). We found five motifs that were well conserved in almost all 82 proteins (Fig. 2a); two upstream motifs of the basic and HLH regions (Fig. $2 \mathrm{~b}$ ), and three motifs at the Cterminus corresponding to the ACT-like domain (Fig. 2c). Some group 1 members, GmbHLH105 and 106 and LjbHLH021, lacked the basic region (Additional file 3: Fig. S3) and these three proteins clustered together in the phylogenetic tree (Additional file 3: Fig. S2).

We confirmed that exon/intron structures are conserved among subclade IVa bHLH genes with some exceptions (Fig. 3). Most members had four exons and three introns. All 82 subclade IVa bHLH genes contained one intron within the HLH domain, but its length was highly variable (Additional file 1: Table S3). This conserved intron position corresponded to pattern $\mathrm{D}$, as defined in a previous study [28]. MtbHLH138, MtbHLH177, GmbHLH334, and LjbHLHO14 lacked intron 3 and exon 4 (Additional file 1: Table S3), resulting in incomplete or absent ACT-like domains (Additional file 3: Fig. S3). As some members of groups 1, 2, and 3 gained or lacked introns (Additional file 1: Table S3), structural diversification may have occurred independently during their evolution.

Based on the highly conserved protein domains and exon-intron organisation across groups, we confirmed that groups 1,2 , and 3 were undoubtedly members of subclade IVa.

\section{Expression patterns of bHLH genes in each group}

Using publicly available expression atlases of G. $\max , M$. truncatula, and L. japonicus, we compared the

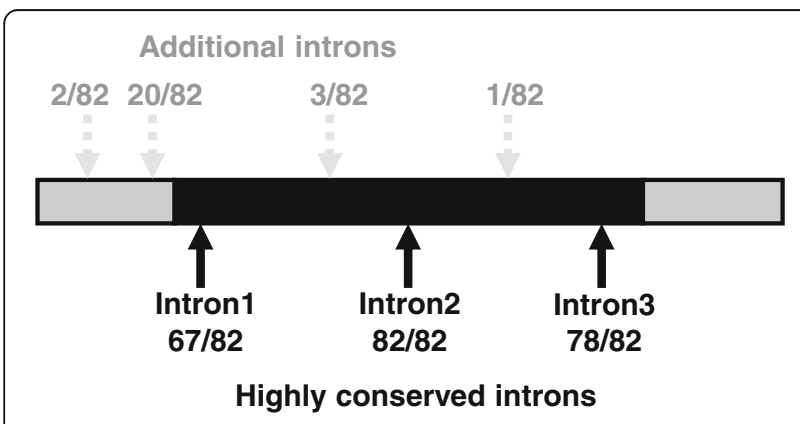

Fig. 3 Simplified exon-intron organisation. Coding sequences (CDSs) and untranslated regions (UTRs) are indicated with black and grey boxes, respectively. Arrows indicate the positions of introns that are well conserved among 82 subclade IVa members from G. max, M. truncatula, and L. japonicus. Grey dashed arrows show the positions of introns found in some subclade IVa members. Details are provided in Additional file 1: Table S3 expression patterns of homologous genes in each plant (Table 2). The orthologous genes in group 1 did not have a completely conserved expression profile across plant species. For instance, although TSAR1 (MtbHLH150) was expressed more in leaves and petioles, the expression levels of its orthologous genes, LjbHLH054 and GmbHLH345, were highest in nodules and flowers, respectively (Additional file 3: Fig. S4). Group 2 members were commonly expressed in nodules, except GmbHLH116 and GmbHLH334, for which expression was not observed. Almost all genes in group 3 were expressed in underground tissues, namely roots and nodules. Three of the four genes in group 3 of $G$. max were also expressed in the pod shells.

\section{Discussion}

One of the most diverse plant transcription factor families, bHLHs regulate many aspects of biological processes, including organ development, specialised metabolism, and the response to environmental stimuli [19]. Subclade IVa bHLH members appear to regulate specialised metabolism and defense responses [5, 19]. In this study, we showed that Fabaceae plants possessed a greater number of subclade IVa bHLH genes in their genomes than other fabids (Table 1, Fig. 1). G. max and Glycine soja had approximately double the number of total bHLHs and subclade IVa members compared to other Fabaceae, as they have experienced two wholegenome duplication events, doubling their genome size $[35,36]$. Although the number of bHLHs in M. truncatula was similar to those of other Fabaceae plants, twice as many subclade IVa bHLHs were found in the Medicago genome (Table 1 ). Thus, $M$. truncatula likely duplicated its subclade IVa bHLHs during development of the hemolytic saponin biosynthesis pathway from the soyasaponin pathway (Additional file 3: Fig. S5).

Domain structures and exon-intron organisation were highly conserved among the 82 subclade IVa members derived from G. $\max , M$. truncatula, and $L$. japonicus (Figs. 2, 3). Fabaceae subclade IVa bHLH proteins were clearly classified into three groups in the phylogenetic tree (Fig. 1). We found a strong bias in the number of Fabaceae bHLHs belonging to group 1, although no such bias was found in other fabids (Table 1). Group 1 may be a clade of transcription factors regulating saponin biosynthesis across a broad range of Fabaceae plants, as all MtTSARs and GubHLH3 were included in this group (Additional file 3: Fig. S2). Furthermore, the expression patterns of orthologous genes in group 1 were not conserved (Table 2), and the soyasaponin biosynthesis regulator, GubHLH3 was not the closest homologue of MtTSAR1 [22]. Thus, although the duplications of group 1 members apparently occurred in ancestral Fabaceae, their expression patterns and contributions to saponin 
Table 2 Tissues expressing subclade IVa bHLHs

\begin{tabular}{|c|c|c|c|}
\hline & Name & Gene ID & Representative tissues expressing the gene \\
\hline \multirow[t]{31}{*}{ Group1 } & TSAR3 homologues & & \\
\hline & GmbHLH100 & Glyma13g44570 & Flower, pod shell (14-17 DAF) \\
\hline & GmbHLH101 & Glyma15g00750 & Flower, pod shell (14-17 DAF) \\
\hline & GmbHLH96 & Glyma08g23060 & Pod shell (7 DAF and 10-13 DAF) \\
\hline & GmbHLH97 & Glyma07g03050 & Young leaf \\
\hline & LjbHLH086 & Lj3g3v0028570 & Not available \\
\hline & & LotjaGi3g1 v0005700 & Immmature flower \\
\hline & MtbHLH070 & Medtr2g104590 & Not available \\
\hline & MtbHLH071/TSAR3 & Medtr2g104650 & $24 d$ seeds \\
\hline & MtbHLH177 & Medtr8g069740 & Not available \\
\hline & TSAR2 homologues & & \\
\hline & GmbHLH118 & Glyma05g23530 & Pod shell \\
\hline & GmbHLH117 & Glyma17g16720 & Root hair, Root \\
\hline & LjbHLH016 & Lj0g3v0151119 & Not available \\
\hline & & LotjaGi4g1 v0240800 & Nodules_10dpi, Root hair \\
\hline & MtbHLH093/TSAR2 & Medtr4g066460 & Not available \\
\hline & & Mtr.9397.1.S1_at & Root, Bacterial and Fungal infections \\
\hline & MtbHLH007 & Medtr0246s0020 & Not available \\
\hline & MtbHLH092 & Medtr4g066380 & Not available \\
\hline & MtbHLH094 & Medtr4g067010 & Not available \\
\hline & MtbHLH004 & Medtr0011s0210 & Not available \\
\hline & MtbHLH005 & Medtr0011s0260 & Not available \\
\hline & MtbHLH009 & Medtr0250s0040 & Not available \\
\hline & MtbHLH139 & Medtr6g047570 & Not available \\
\hline & MtbHLH138 & Medtr6g047550 & Not available \\
\hline & MtbHLH008 & Medtr0246s0050 & Not available \\
\hline & MtbHLH113 & Medtr4g098035 & Not available \\
\hline & TSAR1 homologues & & \\
\hline & GmbHLH345 & Glyma18g48120 & Young leaf, Flower, $1 \mathrm{~cm}$ Pod, Root \\
\hline & LjbHLH054 & Lj1g3v2883900 & Nodule \\
\hline & MtbHLH150/TSAR1 & Medtr7g080780 & Leaf, Root \\
\hline \multirow[t]{8}{*}{ Group2 } & GmbHLH114 & Glyma13g32650 & Nodule, Root, Seeds (10-13 DAF) \\
\hline & GmbHLH115 & Glyma15g06680 & Nodule \\
\hline & GmbHLH116 & Glyma07g30420 & Not expressed \\
\hline & GmbHLH334 & no correspondence & Not available \\
\hline & LjbHLH032 & Lj0g3v0292969 & Root, Nodule \\
\hline & LjbHLH152 & Lj6g3v2171830 & Nodule \\
\hline & MtbHLH043 & Medtr2g010450 & Nodule (4d), Root \\
\hline & MtbHLH107 & Medtr4g092700 & Nodule (4d, 14d, 10d) \\
\hline \multirow[t]{5}{*}{ Group3 } & GmbHLH1 10 & Glyma17g16740 & Pod shells \\
\hline & GmbHLH111 & Glyma05g23290 & Root \\
\hline & GmbHLH112 & Glyma11g04690 & Nodule, pod shell (14-17 DAF) \\
\hline & GmbHLH113 & Glyma01g40600 & Nodule, pod shell (14-17 DAF) \\
\hline & LjbHLH001 ${ }^{a}$ & Lj0g3v0034169 & Not available \\
\hline
\end{tabular}


Table 2 Tissues expressing subclade IVa bHLHs (Continued)

\begin{tabular}{lll}
\hline Name & Gene ID & Representative tissues expressing the gene \\
\hline LjbHLH014 & Lj0g3v0140069 & Not available \\
a & LotjaGi4g1v0185900 & Root \\
LjbHLH081 & Lj2g3v1984450 & Root, Nodule \\
MtbHLH110 & Medtr4g097920 & Nodule (4d) \\
MtbHLH111 & Medtr4g097940 & Nodule (4d) \\
MtbHLH123 & Medtr5g014640 & Nodule (4d) \\
\hline
\end{tabular}

The expression of representative genes belonging to subclade IVa was determined using publicly available databases and summarised. ${ }^{\mathrm{a}} \mathrm{LjbHLH} 001$ and LjbHLH014 are found in the L. japonicus Miyakojima MG-20 accession, but both correspond to the same gene in the L. japonicus Gifu B-129 accession

biosynthesis may have differentiated after speciation. Therefore, we should search for candidate soyasaponin biosynthesis regulators among group 1 members.

Fewer members belonged to groups 2 and 3, but were highly conserved (Fig. 1, Table 1) and tended to be expressed in nodules and roots (Table 2). We confirmed the co-expression of LjCYP93E1 (a soyasaponin biosynthetic gene) and LjbHLHO32 (group 2 subclade IVa bHLH) with a Pearson's correlation coefficient of 0.797 (Additional file 3: Fig. S6). Furthermore, Fabaceae triterpene saponins likely play important roles in the rhizosphere, as reported in previous studies; increased saponin accumulation enhanced nodulation [16] and soyasaponins were the major component of root exudates [37]. These observations suggest that members of group 2 affect biological interactions in the rhizosphere through modulation of soyasaponin production. Generally, bHLH proteins form homo- and heterodimers that regulate the expression of target genes [18, 25, 32, 33]. The possibility that subclade IVa members in groups 2 and 3 also regulate saponin biosynthesis in Fabaceae is worthy of further investigation.

Fabaceae possessed more subclade IVa members, although there was no significant difference in the total numbers of bHLH genes between Fabaceae and non-Fabaceae (MannWhitney $\mathrm{U}$ test, $U=210, p=0.1639$ ). This suggested that other subclades in Fabaceae might have fewer genes. We roughly estimated how many genes were present in each subclade in selected species based on the phylogenetic relationships of the bHLH domains, and found no specific contraction in any subclade (Additional file 1: Table S4).

\section{Conclusions}

In this study, we constructed a phylogenetic tree of fulllength subclade IVa bHLH proteins from 40 plant species, mainly comprised of fabids. The results clearly indicated that subclade IVa bHLHs could be classified into three groups, and that Fabaceae plants contained a large number of group 1 members, including all saponin biosynthesis regulators identified to date. This information will help to uncover unidentified soyasaponin biosynthesis regulatory factors. On the other hand, no genes in groups
2 or 3 have yet been functionally characterised in Fabaceae. These genes are interesting targets for elucidating the evolution and functions of Fabaceae subclade IVa bHLH transcription factors.

\section{Methods}

\section{Sequence retrieval}

Representative protein sequences of G. uralensis were obtained from the G. uralensis genome database [38]. A total of 163 putative bHLH proteins were retrieved based on hidden Markov models (HMMs) of HLH domain (PF00010) downloaded from Pfam 32.0 [39, 40], using HMMER v3.3 software $[41,42]$. The bHLH domain sequences and full-length sequences of bHLH proteins (only the primary isoforms) from other plant species were retrieved from PlantTFDB v5.0 [31, 43]. Subclade IVa members of selected species were identified using a BLAST search against all subclade IVa proteins of $A$. thaliana and G. $\max$ with an e-value threshold of $<1 \mathrm{e}-50$. The bHLH proteins selected are listed in Additional file 2.

\section{Phylogenetic tree analysis}

Protein alignment of full-length bHLHs or bHLH domains was performed using Clustal Omega v1.2.3 [44] with the default settings. A Newick file was generated using FastTree v2.1.10 [45] with the default settings. The phylogenetic tree was visualised from the Newick file using MEGA X [46].

\section{Identification of conserved motifs and exon-intron structures}

The conserved motifs of subclade IVa bHLHs from G. max, L. japonicus, and M. truncatula were predicted using MEME v5.1.1 [34, 47]. Exon-intron structures were retrieved from Phytozome v12.1 [48, 49] and the Legume Information System [50, 51].

\section{Expression pattern analysis}

Expression patterns of bHLH genes were retrieved from Lotus Base [52, 53], Soybean eFP browser [54], Medicago eFP browser [55], and The Medicago truncatula Gene Expression Atlas [56, 57]. 


\section{Supplementary Information}

The online version contains supplementary material available at https://doi. org/10.1186/s12870-021-02887-w.

Additional file 1 Table S1. Numbering of G. max, M. truncatula, and L. japonicus bHLH genes. Table S2. List of species used for phylogenetic tree analysis of subclade IVa bHLHs. Table S3. Exon-intron organisation. Genes with additional introns in their CDSs are indicated in red. The length of these additional introns is given in brackets. Introns within the HLH domain are highlighted in yellow. Table S4. Numbers of genes in each subclade.

Additional file 2 Supplemental Data S1. Protein sequences of 362 subclade IVa bHLHs used for phylogenetic tree analysis.

Additional file $\mathbf{3}$ Fig. S1. Phylogenetic tree of subclade IIIf and IVa bHLH proteins in Glycine max and Arabidopsis thaliana. Fig. S2. Detailed phylogenetic tree of subclade IVa bHLHs in fabids. Fig. S3. Predicted domains of subclade IVa bHLH proteins identified using MEME. Fig. S4. Expression patterns of TSAR1 orthologues. Data were retrieved from Lotus Base, Soybean eFP browser, and Medicago eFP browser. Fig. S5. Biosynthesis pathways for aglycones of soyasaponins and hemolytic saponins from $M$. truncatula. This figure shows representative aglycones of soyasaponins and hemolytic saponins. Cytochrome P450

monooxygenases have been found to oxidise different carbon positions of the $\beta$-amyrin backbone [cytochrome P450 enzymes (positions to be oxidised), ${ }^{*}$ characterised in soybean]. Although the soyasaponin pathway is common among Fabaceae, only Medicago spp. acquired the hemolytic pathway. Fig. S6. Expression of LjCYP93E1 and LjbHLH032. Data retrieved from Lotus Base. Lj1g3v3555800: LjCYP93E1; Lj0g3v0292969: LjbHLH032.

\begin{abstract}
Abbreviations
A. thaliana: Arabidopsis thaliana; bHLH: basic-helix-loop-helix; BLAST: Basic Local Alignment Search Tool; BIS: bHLH iridoid synthesis; C. roseus: Catharanthus roseus; C. quinoa: Chenopodium quinoa; CDS: Coding sequence; G. max: Glycine max; G. soja: Glycine soja; G. uralensis: Glycyrrhiza uralensis; HMM: Hidden Markov model; L. japonicus: Lotus japonicus; M. truncatula: Medicago truncatula; MeJA: Methyl jasmonate; MIA: Monoterpenoid indole alkaloid; TSAR: TRITERPENE SAPONIN BIOSYTHESIS ACTIVATING REGULATOR; TSARL: TSAR-like; UTR: Untranslated region
\end{abstract}

\section{Acknowledgements}

Not applicable.

\section{Authors' contributions}

HSe, and TM conceived and supervised the study; HSu, HSe, and TM designed the researches; HSu performed the analyses; HSu wrote the manuscript; HSe and TM made manuscript revisions. All authors read and approved the final manuscript.

\section{Funding}

This work was supported by the Ministry of Education, Culture, Sports, Science and Technology (MEXT), Japan [JSPS KAKENHI grant Nos. JP19J10245 to HSu, JP2OH02913 to HSe, and JP19H02921 to TM].

\section{Availability of data and materials}

The all data analyzed in this study are available in the publications and the websites cited in Methods section.

\section{Ethics approval and consent to participate}

Not applicable.

\section{Consent for publication}

Not applicable.

\section{Competing interests}

The authors declare that they have no competing interests.
Received: 3 August 2020 Accepted: 10 February 2021

Published online: 23 February 2021

\section{References}

1. Thimmappa R, Geisler K, Louveau T, O'Maille P, Osbourn A. Triterpene biosynthesis in plants. Annu Rev Plant Biol. 2014;65:225-57.

2. Seki H, Tamura K, Muranaka T. P450s and UGTs: key players in the structural diversity of triterpenoid saponins. Plant Cell Physiol. 2015;56(8):1463-71.

3. Singh AK, Kumar SR, Dwivedi $V$, et al. A WRKY transcription factor from Withania somnifera regulates triterpenoid withanolide accumulation and biotic stress tolerance through modulation of phytosterol and defense pathways. New Phytol. 2017;215(3):1115-31.

4. $\mathrm{Xu}$ J, Wu S, Xu Y, et al. Overexpression of BcbZIP134 negatively regulates the biosynthesis of saikosaponins. Plant Cell Tissue Organ Cult. 2019;137: 297-308.

5. Goossens J, Mertens J, Goossens A. Role and functioning of bHLH transcription factors in jasmonate signalling. J Exp Bot. 2017;68(6):1333-47.

6. González-Coloma A, López-Balboa C, Santana O, Reina M, Fraga BM. Triterpene-based plant defenses. Phytochem Rev. 2011;10(2):245-60.

7. Vo NNQ, Fukushima EO, Muranaka T. Structure and hemolytic activity relationships of triterpenoid saponins and sapogenins. J Nat Med. 2017; 71(1):50-8.

8. Vo NNQ, Nomura Y, Muranaka T, Fukushima EO. Structure-activity relationships of pentacyclic triterpenoids as inhibitors of cyclooxygenase and lipoxygenase enzymes. J Nat Prod. 2019;82(12):3311-20.

9. Osbourn AE, Clarke BR, Lunness P, Scott PR, Daniels MJ. An oat species lacking avenacin is susceptible to infection by Gaeumannomyces graminis var. tritici. Physiol Mol Plant Pathol. 1994;45(6):457-67.

10. Shinoda $T$, Nagao $T$, Nakayama $M$, et al. Identification of a triterpenoid saponin from a crucifer, Barbarea vulgaris, as a feeding deterrent to the diamondback moth, Plutella xylostella. J Chem Ecol. 2002;28(3):587-99.

11. Huhman DV, Berhow MA, Sumner LW. Quantification of saponins in aerial and subterranean tissues of Medicago truncatula. J Agric Food Chem. 2005; 53(6):1914-20

12. Berhow MA, Kong SB, Vermillion KE, Duval SM. Complete quantification of group a and group B soyasaponins in soybeans. J Agric Food Chem. 2006; 54(6):2035-44

13. Tamura K, Seki H, Suzuki H, Kojoma M, Saito K, Muranaka T. CYP716A179 functions as a triterpene C-28 oxidase in tissue-cultured stolons of Glycyrrhiza uralensis. Plant Cell Rep. 2017;36(3):437-45.

14. Suzuki H, Fukushima EO, Shimizu Y, et al. Lotus japonicus Triterpenoid profile and characterization of the CYP716A51 and LjCYP93E1 genes involved in their biosynthesis in planta. Plant Cell Physiol. 2019;60(11):2496-509.

15. Seki H, Sawai S, Ohyama K, et al. Triterpene functional genomics in licorice for identification of CYP72A154 involved in the biosynthesis of glycyrrhizin. Plant Cell. 2011;23(11):4112-23.

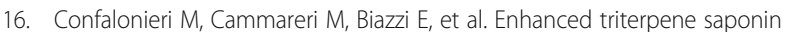
biosynthesis and root nodulation in transgenic barrel medic (Medicago truncatula Gaertn.) expressing a novel beta-amyrin synthase (AsOXA1) gene. Plant Biotechnol J. 2009:7(2):172-82.

17. Delis C, Krokida A, Georgiou S, et al. Role of lupeol synthase in Lotus japonicus nodule formation. New Phytol. 2011;189(1):335-46.

18. Heim MA, Jakoby M, Werber M, Martin C, Weisshaar B, Bailey PC. The basic helix-loop-helix transcription factor family in plants: a genome-wide study of protein structure and functional diversity. Mol Biol Evol. 2003;20(5):735-47.

19. Pires N, Dolan L. Origin and diversification of basic-helix-loop-helix proteins in plants. Mol Biol Evol. 2010;27(4):862-74.

20. Mertens J, Pollier J, Vanden Bossche R, Lopez-Vidriero I, Franco-Zorrilla JM, Goossens A. The bHLH transcription factors TSAR1 and TSAR2 regulate triterpene saponin biosynthesis in Medicago truncatula. Plant Physiol. 2016; 170(1):194-210

21. Ribeiro $B$, Lacchini $E$, Bicalho $K$, et al. A seed-specific regulator of triterpene saponin biosynthesis in Medicago truncatula. Plant Cell. 2020;32(6):2020-42.

22. Tamura K, Yoshida K, Hiraoka Y, et al. The basic helix-loop-helix transcription factor GubHLH3 positively regulates soyasaponin biosynthetic genes in Glycyrrhiza uralensis. Plant Cell Physiol. 2018;59(4):783-96.

23. Jarvis DE, Ho YS, Lightfoot DJ, et al. The genome of Chenopodium quinoa. Nature. 2017;542(7641):307-12.

24. Van Moerkercke A, Steensma P, Schweizer F, et al. The bHLH transcription factor BIS1 controls the iridoid branch of the monoterpenoid indole alkaloid 
pathway in Catharanthus roseus. Proc Natl Acad Sci U S A. 2015;112(26): $8130-5$.

25. Van Moerkercke A, Steensma P, Gariboldi I, et al. The basic helix-loop-helix transcription factor BIS2 is essential for monoterpenoid indole alkaloid production in the medicinal plant Catharanthus roseus. Plant J. 2016;88(1):3-12.

26. Mertens J, Van Moerkercke A, Vanden Bossche R, Pollier J, Goossens A. Clade IVa basic helix-loop-helix transcription factors form part of a conserved jasmonate signaling circuit for the regulation of bioactive plant terpenoid biosynthesis. Plant Cell Physiol. 2016;57(12):2564-75.

27. Fiallos-Jurado J, Pollier J, Moses T, et al. Saponin determination, expression analysis and functional characterization of saponin biosynthetic genes in Chenopodium quinoa leaves. Plant Sci. 2016;250:188-97.

28. Hudson KA, Hudson ME. A classification of basic helix-loop-helix transcription factors of soybean. Int J Genomics. 2015;2015:603182.

29. Sun $\mathrm{H}$, Fan HJ, Ling HQ. Genome-wide identification and characterization of the bHLH gene family in tomato. BMC Genomics. 2015;16(1):9.

30. Zhang C, Feng R, Ma R, et al. Genome-wide analysis of basic helix-loop-helix superfamily members in peach. PLoS One. 2018;13(4):e0195974.

31. Plant transcription factor database. http://planttfdb.cbi.pku.edu.cn/. Accessed 8 May 2020.

32. Feller A, Hernandez JM, Grotewold E. An ACT-like domain participates in the dimerization of several plant basic-helix-loop-helix transcription factors. J Biol Chem. 2006;281(39):28964-74.

33. Kong $Q$, Pattanaik $S$, Feller $A$, et al. Regulatory switch enforced by basic helix-loop-helix and ACT-domain mediated dimerizations of the maize transcription factor R. Proc Natl Acad Sci U S A. 2012;109(30):E2091-7.

34. MEME suite. http://meme-suite.org/index.html. Accessed 16 May 2020.

35. Schmutz J, Cannon SB, Schlueter J, et al. Genome sequence of the palaeopolyploid soybean. Nature. 2010;463(7278):178-83.

36. Xie $M$, Chung CY, Li MW, et al. A reference-grade wild soybean genome. Nat Commun. 2019;10(1):1216.

37. Tsuno Y, Fujimatsu T, Endo K, Sugiyama A, Yazaki K. Soyasaponins: a new class of root exudates in soybean (Glycine max). Plant Cell Physiol. 2018; 59(2):366-75.

38. Mochida K, Sakurai T, Seki H, et al. Draft genome assembly and annotation of Glycyrrhiza uralensis, a medicinal legume. Plant J. 2017:89(2):181-94.

39. El-Gebali S, Mistry J, Bateman A, et al. The Pfam protein families database in 2019. Nucleic Acids Res. 2019:47(D1):D427-32.

40. Pfam. https://pfam.xfam.org/. Accessed 6 May 2020.

41. Finn RD, Clements J, Eddy SR. HMMER web server: interactive sequence similarity searching. Nucleic Acids Res. 2011;39(Web Server issue):W29-37.

42. HMMER. http://hmmer.org/. Accessed 6 May 2020

43. Tian F, Yang DC, Meng YQ, Jin J, Gao G. PlantRegMap: charting functional regulatory maps in plants. Nucleic Acids Res. 2020;48(D1):D1104-13.

44. Sievers F, Wilm A, Dineen D, et al. Fast, scalable generation of high-quality protein multiple sequence alignments using Clustal omega. Mol Syst Biol. 2011;7:539.

45. Price MN, Dehal PS, Arkin AP. FastTree 2--approximately maximumlikelihood trees for large alignments. PLoS One. 2010;5(3):e9490.

46. Kumar S, Stecher G, Li M, Knyaz C, Tamura K. MEGA X: molecular evolutionary genetics analysis across computing platforms. Mol Biol Evol. 2018;35(6):1547-9.

47. Bailey TL, Elkan C. Fitting a mixture model by expectation maximization to discover motifs in biopolymers. Proc Int Conf Intell Syst Mol Biol. 1994;2:2836.

48. Goodstein DM, Shu S, Howson R, et al. Phytozome: a comparative platform for green plant genomics. Nucleic Acids Res. 2012;40(Database issue): D1178-86.

49. Phytozome v12.1. https://phytozome.jgi.doe.gov/pz/portal.html. Accessed 18 May 2020.

50. Dash S, Campbell JD, Cannon EK, et al. Legume information system (Legumelnfo.org): a key component of a set of federated data resources for the legume family. Nucleic Acids Res. 2016;44(D1):D1181-8.

51. Legume information system. https://legumeinfo.org/. Accessed 18 May 2020.

52. Mun T, Bachmann A, Gupta V, Stougaard J, Andersen SU. Lotus Base: an integrated information portal for the model legume Lotus japonicus. Sci Rep. 2016;6:39447.

53. Lotus Base. https://lotus.au.dk/. Accessed 25 May 2020.

54. Soybean eFP browser. http://bar.utoronto.ca/efpsoybean/cgi-bin/efpWeb. cgi. Accessed 19 May 2020.
55. Medicago eFP browser. http://bar.utoronto.ca/efpmedicago/cgi-bin/efpWeb. cgi. Accessed 19 May 2020

56. He J, Benedito VA, Wang M, et al. The Medicago truncatula gene expression atlas web server. BMC Bioinformatics. 2009;10:441.

57. Medicago truncatula Gene Expression Atlas. https://mtgea.noble.org/v3/. Accessed 20 May 2020.

\section{Publisher's Note}

Springer Nature remains neutral with regard to jurisdictional claims in published maps and institutional affiliations.

\section{Ready to submit your research? Choose BMC and benefit from:}

- fast, convenient online submission

- thorough peer review by experienced researchers in your field

- rapid publication on acceptance

- support for research data, including large and complex data types

- gold Open Access which fosters wider collaboration and increased citations

- maximum visibility for your research: over $100 \mathrm{M}$ website views per year

At BMC, research is always in progress.

Learn more biomedcentral.com/submissions 